\title{
Validation of a windowing protocol for accurate in vivo tooth segmentation using i-CAT cone beam computed tomography
}

\author{
Babak Rastegar 1,2,A-F, Brice Thumilaire ${ }^{3, B-F}$, Guillaume A. Odri ${ }^{4,-F}$, Sergio Siciliano ${ }^{5, D-F}$, \\ Jan Zapała ${ }^{6, D-F}$, Pierre Mahy ${ }^{7, D-F}$, Raphael Olszewski ${ }^{7,8, A-F}$ \\ ${ }^{1}$ Service de Parodontologie, Université libre de Bruxelles, Brussels, Belgium \\ 2 PhD student, Jagiellonian University Medical College, Kraków, Poland \\ ${ }^{3}$ Private practice, Libramont, Belgium \\ ${ }^{4}$ Service de Chirurgie Orthopédique et Traumatologique, CHU Lariboisière, Paris, France \\ ${ }^{5}$ Service de Stomatologie et de Chirurgie Maxillo-faciale, Clinique sainte Elisabeth, Brussels, Belgium \\ ${ }^{6}$ Department of Cranio-Maxillofacial Surgery, Jagiellonian University Medical College, Rydygier Hospital, Kraków, Poland \\ ${ }^{7}$ Service de Stomatologie et de Chirurgie Maxillo-faciale, Cliniques Universitaires Saint Luc, Université Catholique de Louvain, Brussels, Belgium \\ ${ }^{8}$ Visiting professor, Department of Cranio-Maxillofacial Surgery, Jagiellonian University Medical College, Rydygier Hospital, Kraków, Poland \\ $A$ - research concept and design; $B$ - collection and/or assembly of data; $C$ - data analysis and interpretation; \\ $D$ - writing the article; $E$ - critical revision of the article; $F$ - final approval of the article
}

Address for correspondence

Raphael Olszewski

E-mail: raphael.olszewski@uclouvain.be

\section{Funding sources}

We would like to thank the Fondation saint Luc, Cliniques Universitaires Saint Luc, for awarding a post-doctoral scholarship to Prof. Raphael Olszewski in 2008 to study 3D Slicer software at the Surgical Planning Lab, Harvard Medical School, Boston, MA, USA.

Conflict of interest None declared

\section{Acknowledgements}

We would like to thank Mr. B. Konieczny and Prof. J. Sokołowski from the Department of Dental Materials at the Medical University of Lodz, Poland, for their help with tooth laser scanning.

Received on September 29, 2016 Reviewed on December 30, 2016 Accepted on December 28, 2016

DOI

10.17219/acem/68117

\section{Copyright}

Copyright by Author(s)

This is an article distributed under the terms of the Creative Commons Attribution Non-Commercial License (http://creativecommons.org/licenses/by-nc-nd/4.0/)

\begin{abstract}
Background. Validation of three-dimensional (3D) reconstructions of full dental arches with crowns and roots based on cone beam computed tomography (CBCT) imaging represents a key issue in 3D digital dentistry.

Objectives. The aim of the study was to search for the most accurate in vivo windowing-based manual tooth segmentation using CBCT. The null hypothesis was that all applied windowing protocols were equivalent in terms of in vivo tooth volume measurement using CBCT.

Material and methods. This retrospective study was based on preoperative CBCT images from patients who underwent further tooth extractions for reasons independent of this study. Written informed consent was obtained from all the participants, and the study was approved by the Ethics Committee of Cliniques Universitaires Saint Luc (Brussels, Belgium). The radiological protocol was I-CAT CBCT, $0.3 \mathrm{~mm}$ slice thickness, $8 \mathrm{~cm} \times 16 \mathrm{~cm}$ field of view, $120 \mathrm{kVp}$, and $18 \mathrm{mAs}$. A total of 36 teeth were extracted from 14 patients between the ages of 18 and 68 years. Using 3D Slicer software, segmentations were performed twice by 2 independent observers, with a 1-month time period between the 2 segmentations to study intra- and inter-observer repeatability and reproducibility. Four windowing protocols (level/window) were applied: 1. 1131/1858, 2. 2224/4095, 3. 1131/4095, and 4. AUT0, an automatic protocol provided by default by the software. A total of 576 segmentations were performed. Tooth volumes were automatically calculated using the software. To compare the volumes obtained from CBCT segmentations with a gold-standard method, we laser-scanned the extracted teeth.
\end{abstract}

Results. Excellent intra- and inter-observer intraclass correlations were found for all of the protocols used. The best windowing protocol was 1131/1858 for both observers. Tooth volumes were obtained by manual segmentation of the CBCT images and using windowing protocol 1131/1858. No significantly different tooth volumes were found by laser scanning.

Conclusions. Our null hypothesis was rejected. Only windowing protocol 1131/1858 allowed for significantly closer 3D in vivo segmentation of a tooth compared to I-CAT CBCT, with excellent intra-observer repeatability and inter-observer reproducibility.

Key words: cone-beam computed tomography, grey levels, segmentation, 3-dimensional 
Validation of 3-dimensional (3D) reconstruction of full dental arches with crowns and roots based on cone beam computed tomography (CBCT) imaging represents a key issue for 3D digital dentistry. Accurate 3D tooth reconstruction allows the construction of individual virtual orthodontic set-ups, roots resorption analysis, virtual planning for mini-implants, and mini-screw insertion. ${ }^{1-4}$ Accurate 3D tooth reconstruction is important when using $3 \mathrm{D}$ printed tooth replicas during autotransplantation procedures, and in custom-made anatomic implantology. ${ }^{5-8}$ Moreover, in forensic odontology accurate tooth volume measurements are important for calculating the tooth/pulp volume ratio, which is strongly correlated with age estimation. ${ }^{9}$ A few studies have already tried to determine and validate in vivo tooth volumes from available $3 \mathrm{D}$ CBCT using manual segmentation, which still represents the gold standard for obtaining volume measurements from medical images. ${ }^{10,11,13-16}$ The first was a 2010 article by Liu et al., who described a feasibility study of in vivo tooth volume determination from CBCT imaging. However, that article had various methodological issues. The authors compared the volume of teeth before extraction (on CBCT), and after extraction (with water displacement as the reference method) on 24 premolars (which represented limited complexity of root anatomy) from 9 patients, using 2 different CBCT devices and 4 different radiological protocols. ${ }^{10}$ Two independent observers manually segmented the 24 premolars twice with Amira 3D software (FEI Visualization Sciences Group, Hillsboro, USA), applying subjective modifications of the windowing of CBCT images during the segmentation process. The reference method was based on water displacement: A subjective visual assessment of the lower level of meniscus ( $0.1 \mathrm{~mL}$ gradation) was performed when the tooth was placed in a graduated cylinder. ${ }^{10}$ Liu et al. concluded that there was a significant difference between the physical volume measurements of the extracted teeth and the CBCT measurements $(-4 \%$ to $+7 \%$; $<<0.05)$. The authors also stated that surface smoothing reduced the volume by $3-12 \%$, and that no specifications could be given at that time regarding how to accurately segment teeth from CBCT. ${ }^{10}$ In a 2011 study by Wang et al., 27 premolars from 15 patients were CBCT-scanned before extraction and micro-CT-scanned after extraction as a comparative reference system..${ }^{11}$ A unique threshold (1673 to $2000 \mathrm{HU}$ ) was employed using Mimics software (Materialise NV, Leuven, Belgium) for tooth segmentation. Intra-observer repeatability was high for both observers (intraclass correlation coefficient $[\mathrm{ICC}]=0.999$ and 0.998 ), while inter-observer reproducibility was less impressive $(\mathrm{ICC}=0.740) .{ }^{11}$ The ICC is used for quantitative measurements of units organized into groups. It describes how strongly units in the same group resemble each other. ${ }^{12}$ The effect of the threshold on segmentation was not the purpose of that study. ${ }^{11}$ Forst et al. proposed to test the intra-and inter-observer reproducibility of 3 types of in vivo segmentation on 10 maxillary upper molars from CBCT: 1. manual human segmentation on a repeated 2-dimensional (2D) basis, 2. automated segmentation without human refinement, and 3. automated segmentation with manual human refinement on a repeated $2 \mathrm{D}$ basis. ${ }^{13}$ The results of automated segmentation with human refinement corresponded to the results of manual segmentation by Liu et al. ${ }^{10}$ However, because the teeth were not extracted after CBCT in that study, it was impossible to determine the true tooth volume using any of the existing reference methods (micro-CT scan, laser scanning, water displacement method, etc.). ${ }^{13}$ The segmentation method proposed by Forst et al. still needs to be validated. The windowing protocol for manual segmentation was not described or tested in that article. ${ }^{13}$ Moreover, some windowing protocols (center level/band width) have been cited in the literature as providing the best tooth visualization in CBCT. Spin-Neto et al. suggested a 1131/1858 protocol for the best tooth visualization. ${ }^{17}$ Lee et al. used a 2224/4095 protocol to generate 3D virtual surface models of the maxillary and mandibular dental arches that include whole with the roots. ${ }^{18}$ However, the protocols by Spin-Neto et al. and Lee et al. have not been validated. Therefore, we proposed to search for the potentially most accurate in vivo windowing-based tooth segmentation protocol from CBCT. We wanted to compare the 2 protocols found in the literature with 2 proposed by our research team: 1 . automatic adjustment of the windowing by the 3D Slicer software AUTO protocol, and 2. a mixed approach based on the protocols found in the literature: $1131 / 4095 .^{17,18}$ The null hypothesis was that all 4 windowing protocols were equivalent for in vivo tooth volume measurement from CBCT.

\section{Material and methods}

This retrospective study was conducted between 2010 and 2014, on the basis of preoperative CBCT examinations of patients who underwent further tooth extractions for reasons independent of the present study. Preoperative CBCTs were also performed independently of this study. Written informed consent was obtained from all the participants in the study, which was approved by the Ethics Committee of Cliniques Universitaires Saint Luc (Brussels, Belgium; no. B40320096961). The radiological protocol was I-CAT (Imaging Sciences International Inc., Hatfield, USA), with $0.3 \mathrm{~mm}$ slice thickness, field of view $8 \mathrm{~cm}$ in height $\times 16 \mathrm{~cm}$ in diameter, $120 \mathrm{kVp}$ and $18 \mathrm{mAs}$. The main reasons for performing CBCT were: 1 . to evaluate the distance between the roots of the wisdom teeth and the inferior alveolar nerve when a close relationship was seen on previously obtained panoramic $\mathrm{X}$-rays; 2 . to evaluate the positioning of supranumerary teeth when 2D X-rays failed to provide a final diagnosis; and 3. to evaluate cystic involvement in the maxillary sinus region associated with maxillary tooth extraction. For the purpose of our study, we used all the extracted teeth that were fully present in the CBCT field of view. For example, 
if CBCT was performed to evaluate the distance between the lower wisdom teeth roots and the inferior alveolar nerve, and simultaneously, the upper wisdom teeth were present in the same CBCT field of view, and were extracted at the same time as the lower wisdom teeth, then the upper wisdom teeth were also used in our study. The inclusion criteria were: adult patients, teeth without demineralization (without decay and without immature roots), teeth without metallic fillings or crowns, and teeth that were not sectioned or damaged during oral surgery. The 36 teeth included were extracted from 14 patients, aged between 18-68 years old. Ten females and 4 males participated in this study. Out of the 36 teeth, 23 were wisdom teeth. Each extracted tooth was first cleaned of blood, soft tissue and bone fragments, and brushed under running water. Next, each tooth was separately conserved in a closed storage box, fully immersed in $0.1 \%$ chloramine.

To study intra- and inter-observer reproducibility, manual segmentations were performed twice for each of the 36 teeth from CBCT imaging by 2 independent observers, with a 1-month time period between the 2 segmentations. Both observers were dentists, and a calibration session was provided by the senior researcher prior to the start of the study to explain how the software worked, how to save the data, how to use different segmentation tools and how to modify the windowing protocols. Prior to the start of the study, the two observers had trained with manual segmentation on 10 other teeth that were then excluded from the main study. The segmentations were performed using 3D Slicer 3.6.1 open source software (SPL, Harvard Medical School, Boston, USA). ${ }^{19}$ Each segmentation consisted of a contour of the tooth image (enamel, dentin, crown and roots), sliceby-slice, by looking at the slices on the computer screen, using a mouse, and using the Editor module of the 3D Slicer software tools, with such functions as: 1. "paint", 2. "level tracing" and 3. "draw". The segmentations were mainly performed on axial slices, and some adjustments were then performed on sagittal and coronal views (Fig. 1). Four different windowing protocols (level/window) were applied: 1. $1131 / 1858,{ }^{16} 2.2224 / 4095,{ }^{18} 3$. a mixed protocol based on protocols 1 and 2:1131/4095, and 4. AUTO, an automatic protocol provided by default by the software (Fig. 2). In total, 576 segmentations were performed. Three-dimensional reconstruction of each tooth was automatically performed using 3D Slicer software, and the tooth volume was automatically calculated by the software. No smoothing function was used, as smoothing may modify the entire volume of the tooth. ${ }^{10}$ The volume was also automatically measured in $\mathrm{mm}^{3}$ using the Statistics module of the 3D Slicer software. To compare the volumes obtained from CBCT segmentations with a gold-standard method, we used the laser scanning method (Ceramill Map300, Amann Girrbach AG, Koblach, Austria), which is based on structural light. The 36 teeth were laser-scanned according to the manufacturer's instructions, within an error of $20 \mu \mathrm{m}$ (Tables 1-4). Prior to the start of the measurements with the laser scanner,

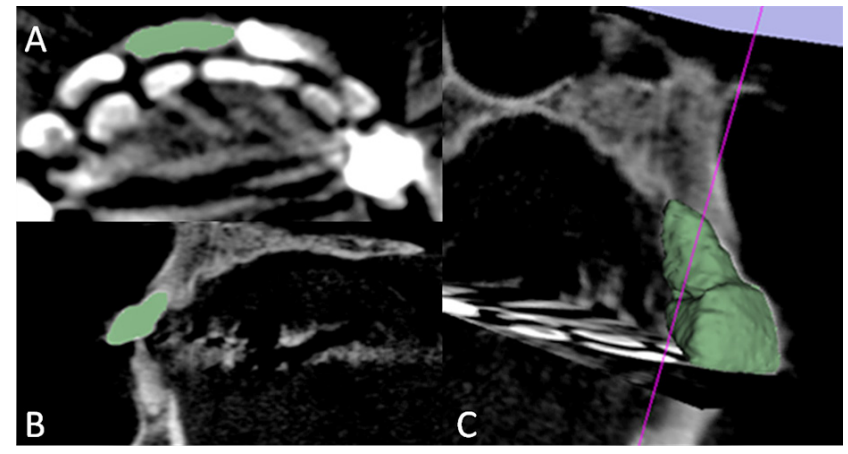

Fig. 1. Manual segmentation of tooth number 11 with 3D Slicer software

A - segmentation on axial slice; B - segmentation on sagittal slice; $\mathrm{C}$ - three-dimensional reconstruction and automatic volume measurement.

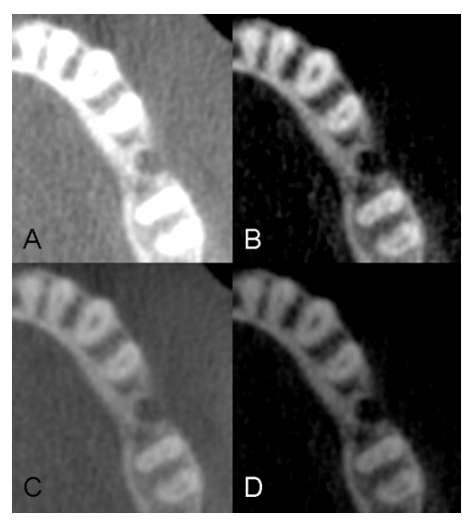

Fig. 2. Four windowing protocols applied to the same single axial slice from I-CAT cone beam computed tomography

A - AUTO; B - 1131/1858; C- 1131/4095; D - 2224/4095.

the device was calibrated using a 3D model provided by the manufacturer. A plaster custom-made support was created for each tooth. The tooth was attached to the support using dental wax. All the surfaces of the tooth were covered with a very thin layer of talc used in the spray for laser scanning purposes (Lava ${ }^{\mathrm{TM}}$, 3M ESPE AG, Seefeld, Germany). The volume was automatically measured by the software included with the laser scanner, and was rechecked with Netfabb basic software (Netfabb GmbH, Parsburg, Germany). Laser scanning one tooth took approx. 40-80 min.

\section{Statistical analysis}

To compare the $1^{\text {st }}$ and $2^{\text {nd }}$ measurements for each windowing protocol, we used multiple paired Student's t-tests with the Bonferroni correction; the level of significance was set at $\mathrm{p}<0.00625$ for intra-observer correlations ( $\mathrm{p}<0.05 / 8$, because there were 8 tests) and $\mathrm{p}<0.0125$ for inter-observer correlations ( $\mathrm{p}<0.05 / 4$, because there were 4 tests). To determine the reproducibility of measurements, we used the intraclass correlation coefficient ICC2.1 (single measurement, absolute agreement, 2-way random effect analysis of variance model). To compare manually segmented volumes from CBCT and the true volume obtained by laser scanner, we again used multiple paired Student's t-tests with the Bonferroni correction; the level of significance was again set at $\mathrm{p}<0.00625$. The correlation 
Table 1. Comparisons between the true volumes of teeth measured by laser scanner and the volumes obtained by 2 observers using the AUTO windowing protocol twice. The measurements are in $\mathrm{mm}^{3}$

\begin{tabular}{|c|c|c|c|c|c|c|}
\hline \multirow[b]{2}{*}{ Tooth } & \multirow{2}{*}{$\begin{array}{l}\text { Type of } \\
\text { tooth }\end{array}$} & \multirow{2}{*}{$\begin{array}{c}\text { True } \\
\text { volume }\end{array}$} & \multicolumn{4}{|c|}{ AUTO } \\
\hline & & & $\begin{array}{c}\text { Obs } 1 \\
1^{\text {st }}\end{array}$ & $\begin{array}{c}\text { Obs } 1 \\
2^{\text {nd }}\end{array}$ & $\begin{array}{c}\text { Obs } 2 \\
1^{\text {st }}\end{array}$ & $\begin{array}{l}\text { Obs } 2 \\
2^{\text {nd }}\end{array}$ \\
\hline 1 & 18 & 830 & 876.71 & 888.20 & 964.83 & 767.43 \\
\hline 2 & 18 & 490 & 520.83 & 539.17 & 524.61 & 513.65 \\
\hline 3 & 28 & 770 & 806.37 & 798.06 & 858.10 & 850.21 \\
\hline 4 & 18 & 630 & 639.32 & 645.30 & 659.11 & 638.40 \\
\hline 5 & 28 & 580 & 591.07 & 621.53 & 638.05 & 649.50 \\
\hline 6 & 18 & 590 & 612.21 & 618.81 & 643.48 & 647.39 \\
\hline 7 & 28 & 710 & 767.08 & 751.30 & 803.21 & 807.74 \\
\hline 8 & 28 & 910 & 908.09 & 915.27 & 910.61 & 920.92 \\
\hline 9 & 18 & 820 & 744.22 & 914.88 & 839.74 & 846.57 \\
\hline 10 & 18 & 560 & 579.78 & 599.07 & 610.78 & 612.10 \\
\hline 11 & 46 & 860 & 872.49 & 875.36 & 986.75 & 1024.74 \\
\hline 12 & 28 & 850 & 960.04 & 932.26 & 973.20 & 980.92 \\
\hline 13 & 18 & 1150 & 1207.30 & 1187.00 & 1214.36 & 1194.70 \\
\hline 14 & 18 & 510 & 549.26 & 532.61 & 548.65 & 536.72 \\
\hline 15 & 12 & 320 & 346.91 & 372.51 & 395.03 & 412.20 \\
\hline 16 & 13 & 590 & 600.49 & 714.89 & 642.42 & 719.89 \\
\hline 17 & 14 & 420 & 444.81 & 501.92 & 453.21 & 497.62 \\
\hline 18 & 34 & 350 & 380.96 & 401.26 & 411.98 & 413.44 \\
\hline 19 & 44 & 350 & 372.94 & 396.94 & 395.59 & 374.23 \\
\hline 20 & 45 & 300 & 297.59 & 293.46 & 303.31 & 320.64 \\
\hline 21 & 18 & 590 & 662.71 & 623.04 & 695.10 & 685.76 \\
\hline 22 & 28 & 680 & 762.59 & 756.48 & 803.96 & 765.38 \\
\hline 23 & 11 & 490 & 655.84 & 606.86 & 659.07 & 654.99 \\
\hline 24 & 12 & 350 & 458.88 & 374.92 & 452.74 & 430.08 \\
\hline 25 & 13 & 460 & 596.74 & 528.92 & 656.59 & 626.83 \\
\hline 26 & 21 & 530 & 625.24 & 580.27 & 629.51 & 623.97 \\
\hline 27 & 22 & 270 & 306.21 & 297.36 & 310.53 & 293.84 \\
\hline 28 & 23 & 630 & 750.39 & 703.54 & 747.25 & 744.55 \\
\hline 29 & 28 & 790 & 835.56 & 762.92 & 838.76 & 797.07 \\
\hline 30 & 38 & 900 & 1045.50 & 839.63 & 997.55 & 975.00 \\
\hline 31 & 38 & 930 & 1014.50 & 1050.50 & 1032.22 & 1013.61 \\
\hline 32 & 28 & 750 & 826.42 & 847.08 & 895.81 & 872.49 \\
\hline 33 & 28 & 710 & 799.70 & 812.23 & 830.33 & 842.54 \\
\hline 34 & 38 & 830 & 878.43 & 903.59 & 901.48 & 890.90 \\
\hline 35 & 48 & 720 & 779.17 & 784.13 & 790.02 & 805.71 \\
\hline 36 & 18 & 560 & 579.01 & 584.98 & 583.19 & 570.20 \\
\hline
\end{tabular}

was calculated between the volume obtained by manual segmentation according to the type of windowing protocol and the true volume measured by the laser scan. A correlation was described as strong if the ICC was greater than 0.8 . All the tests were performed using $\mathrm{SPSS}^{\circledR}$ for Windows, v. 16.0 (SPSS Inc., Chicago, USA).
Table 2. Comparisons between the true volumes of teeth measured by laser scanner and the volumes obtained by 2 observers using the $1131 / 1858$ windowing protocol twice. The measurements are in $\mathrm{mm}^{3}$

\begin{tabular}{|c|c|c|c|c|c|c|}
\hline \multirow[b]{2}{*}{ Tooth } & \multirow{2}{*}{$\begin{array}{l}\text { Type of } \\
\text { tooth }\end{array}$} & \multirow{2}{*}{$\begin{array}{c}\text { True } \\
\text { volume }\end{array}$} & \multicolumn{4}{|c|}{$1131 / 1858$} \\
\hline & & & $\begin{array}{c}\text { Obs } 1 \\
1^{\text {st }}\end{array}$ & $\begin{array}{l}\text { Obs } 1 \\
2^{\text {nd }}\end{array}$ & $\begin{array}{c}\text { Obs } 2 \\
1^{\text {st }}\end{array}$ & $\begin{array}{l}\text { Obs } 2 \\
2^{\text {nd }}\end{array}$ \\
\hline 1 & 18 & 830 & 812.84 & 821.35 & 781.71 & 861.07 \\
\hline 2 & 18 & 490 & 495.80 & 492.39 & 440.75 & 474.01 \\
\hline 3 & 28 & 770 & 703.14 & 756.56 & 726.61 & 863.06 \\
\hline 4 & 18 & 630 & 639.32 & 645.30 & 659.11 & 638.40 \\
\hline 5 & 28 & 580 & 565.07 & 596.51 & 459.57 & 577.92 \\
\hline 6 & 18 & 590 & 574.13 & 567.44 & 526.16 & 591.53 \\
\hline 7 & 28 & 710 & 668.17 & 698.85 & 654.44 & 742.38 \\
\hline 8 & 28 & 910 & 912.44 & 868.10 & 828.26 & 926.59 \\
\hline 9 & 18 & 820 & 788.81 & 808.79 & 774.18 & 837.33 \\
\hline 10 & 18 & 560 & 519.74 & 524.69 & 558.18 & 550.14 \\
\hline 11 & 46 & 860 & 729.98 & 773.71 & 740.40 & 812.46 \\
\hline 12 & 28 & 850 & 875.04 & 886.71 & 865.41 & 848.32 \\
\hline 13 & 18 & 1150 & 1103.30 & 1132.06 & 1130.68 & 1162.84 \\
\hline 14 & 18 & 510 & 514.96 & 521.46 & 487.55 & 539.37 \\
\hline 15 & 12 & 320 & 325.27 & 323.59 & 350.67 & 288.00 \\
\hline 16 & 13 & 590 & 579.35 & 558.31 & 561.12 & 487.28 \\
\hline 17 & 14 & 420 & 419.70 & 405.73 & 389.47 & 331.41 \\
\hline 18 & 34 & 350 & 356.47 & 356.85 & 379.64 & 304.25 \\
\hline 19 & 44 & 350 & 358.53 & 342.88 & 359.34 & 294.78 \\
\hline 20 & 45 & 300 & 279.74 & 267.46 & 296.36 & 203.36 \\
\hline 21 & 18 & 590 & 590.67 & 587.95 & 582.84 & 603.57 \\
\hline 22 & 28 & 680 & 702.10 & 673.09 & 668.94 & 669.21 \\
\hline 23 & 11 & 490 & 560.34 & 546.17 & 485.59 & 559.95 \\
\hline 24 & 12 & 350 & 381.72 & 345.23 & 313.66 & 378.75 \\
\hline 25 & 13 & 460 & 440.54 & 476.65 & 447.71 & 529.20 \\
\hline 26 & 21 & 530 & 532.49 & 556.71 & 503.87 & 574.48 \\
\hline 27 & 22 & 270 & 263.44 & 267.86 & 236.06 & 289.49 \\
\hline 28 & 23 & 630 & 671.54 & 642.07 & 568.92 & 689.99 \\
\hline 29 & 28 & 790 & 670.78 & 663.75 & 748.17 & 675.41 \\
\hline 30 & 38 & 900 & 933.11 & 781.05 & 764.45 & 787.67 \\
\hline 31 & 38 & 930 & 977.14 & 1048.90 & 948.00 & 963.12 \\
\hline 32 & 28 & 750 & 774.10 & 741.15 & 684.84 & 756.60 \\
\hline 33 & 28 & 710 & 622.50 & 647.84 & 609.42 & 784.92 \\
\hline 34 & 38 & 830 & 774.91 & 783.32 & 753.76 & 819.21 \\
\hline 35 & 48 & 720 & 681.24 & 704.02 & 644.25 & 763.08 \\
\hline 36 & 18 & 560 & 545.97 & 502.08 & 519.41 & 593.07 \\
\hline
\end{tabular}

\section{Results}

Table 5 shows the intra-observer correlations for the 2 observers in relation to the 4 different windowing protocols. There were no significant differences between the $1^{\text {st }}$ and $2^{\text {nd }}$ measurements. There was a good intraclass correlation for all of the protocols used. The means of the $1^{\text {st }}$ and $2^{\text {nd }}$ measurements were then used for further tests. 
Table 3. Comparisons between the true volumes of teeth measured by laser scanner and the volumes obtained by 2 observers using the $2224 / 4095$ windowing protocol twice. The measurements are in $\mathrm{mm}^{3}$

\begin{tabular}{|c|c|c|c|c|c|c|}
\hline \multirow[b]{2}{*}{ Tooth } & \multirow{2}{*}{$\begin{array}{l}\text { Type of } \\
\text { tooth }\end{array}$} & \multirow{2}{*}{$\begin{array}{c}\text { True } \\
\text { volume }\end{array}$} & \multicolumn{4}{|c|}{$2224 / 4095$} \\
\hline & & & $\begin{array}{l}\text { Obs } 1 \\
1^{\text {st }}\end{array}$ & $\begin{array}{l}\text { Obs } 1 \\
2^{\text {nd }}\end{array}$ & $\begin{array}{l}\text { Obs } 2 \\
1^{\text {st }}\end{array}$ & $\begin{array}{c}\text { Obs } 2 \\
2^{\text {nd }}\end{array}$ \\
\hline 1 & 18 & 830 & 781.02 & 815.64 & 767.43 & 821.67 \\
\hline 2 & 18 & 490 & 477.70 & 460.26 & 396.60 & 430.57 \\
\hline 3 & 28 & 770 & 701.50 & 716.47 & 703.66 & 752.53 \\
\hline 4 & 18 & 630 & 596.34 & 580.47 & 579.27 & 595.71 \\
\hline 5 & 28 & 580 & 559.58 & 529.92 & 545.74 & 565.42 \\
\hline 6 & 18 & 590 & 556.45 & 543.12 & 523.17 & 544.47 \\
\hline 7 & 28 & 710 & 662.02 & 661.58 & 681.73 & 679.55 \\
\hline 8 & 28 & 910 & 846.81 & 787.04 & 788.13 & 828.42 \\
\hline 9 & 18 & 820 & 778.60 & 776.70 & 766.54 & 745.18 \\
\hline 10 & 18 & 560 & 507.03 & 519.70 & 521.79 & 519.17 \\
\hline 11 & 46 & 860 & 786.28 & 776.82 & 768.61 & 720.64 \\
\hline 12 & 28 & 850 & 876.79 & 835.67 & 859.63 & 794.40 \\
\hline 13 & 18 & 1150 & 1087.80 & 1096.10 & 1125.50 & 1071.26 \\
\hline 14 & 18 & 510 & 478.58 & 494.05 & 479.80 & 467.87 \\
\hline 15 & 12 & 320 & 314.44 & 302.51 & 285.49 & 258.89 \\
\hline 16 & 13 & 590 & 582.47 & 545.13 & 501.67 & 464.14 \\
\hline 17 & 14 & 420 & 406.77 & 388.00 & 354.66 & 336.11 \\
\hline 18 & 34 & 350 & 313.70 & 329.41 & 315.64 & 253.12 \\
\hline 19 & 44 & 350 & 336.86 & 331.32 & 335.49 & 263.86 \\
\hline 20 & 45 & 300 & 260.19 & 254.73 & 239.49 & 190.75 \\
\hline 21 & 18 & 590 & 580.50 & 570.66 & 565.77 & 564.80 \\
\hline 22 & 28 & 680 & 648.11 & 652.61 & 652.68 & 632.51 \\
\hline 23 & 11 & 490 & 582.23 & 493.28 & 470.77 & 502.55 \\
\hline 24 & 12 & 350 & 354.87 & 322.98 & 309.80 & 331.83 \\
\hline 25 & 13 & 460 & 436.18 & 488.34 & 461.86 & 464.29 \\
\hline 26 & 21 & 530 & 500.24 & 527.38 & 499.61 & 526.88 \\
\hline 27 & 22 & 270 & 236.80 & 262.04 & 231.31 & 262.33 \\
\hline 28 & 23 & 630 & 595.12 & 593.16 & 544.73 & 600.99 \\
\hline 29 & 28 & 790 & 690.23 & 700.29 & 676.49 & 675.87 \\
\hline 30 & 38 & 900 & 851.05 & 730.93 & 793.53 & 754.25 \\
\hline 31 & 38 & 930 & 991.08 & 974.34 & 917.73 & 838.27 \\
\hline 32 & 28 & 750 & 750.85 & 747.15 & 672.99 & 742.86 \\
\hline 33 & 28 & 710 & 612.83 & 633.57 & 605.29 & 661.20 \\
\hline 34 & 38 & 830 & 745.58 & 748.93 & 736.21 & 751.47 \\
\hline 35 & 48 & 720 & 652.53 & 674.17 & 654.16 & 674.65 \\
\hline 36 & 18 & 560 & 513.32 & 461.85 & 501.89 & 531.43 \\
\hline
\end{tabular}

Table 6 shows the inter-observer correlations for the 2 observers in relation to the 4 different windowing protocols. The inter-observer correlation was excellent for all 4 windowing protocols. There was a significant difference between the 2 observers for the AUTO and 2224/4095 protocols. However, this error is small compared to the measured volume (excellent ICC).
Table 4. Comparisons between the true volumes of teeth measured by laser scanner and the volumes obtained by 2 observers using the $1131 / 4095$ windowing protocol twice. The measurements are in $\mathrm{mm}^{3}$

\begin{tabular}{|c|c|c|c|c|c|c|}
\hline \multirow[b]{2}{*}{ Tooth } & \multirow{2}{*}{$\begin{array}{l}\text { Type of } \\
\text { tooth }\end{array}$} & \multirow{2}{*}{$\begin{array}{c}\text { True } \\
\text { volume }\end{array}$} & \multicolumn{4}{|c|}{$1131 / 4095$} \\
\hline & & & $\begin{array}{c}\text { Obs } 1 \\
1^{\text {st }}\end{array}$ & $\begin{array}{l}\text { Obs } 1 \\
2^{\text {nd }}\end{array}$ & $\begin{array}{c}\text { Obs } 2 \\
1^{\text {st }}\end{array}$ & $\begin{array}{l}\text { Obs } 2 \\
2^{\text {nd }}\end{array}$ \\
\hline 1 & 18 & 830 & 769.40 & 824.60 & 753.72 & 868.49 \\
\hline 2 & 18 & 490 & 485.01 & 473.10 & 441.56 & 435.35 \\
\hline 3 & 28 & 770 & 709.14 & 751.61 & 705.44 & 835.79 \\
\hline 4 & 18 & 630 & 592.90 & 596.51 & 570.28 & 581.40 \\
\hline 5 & 28 & 580 & 563.01 & 545.85 & 538.99 & 559.99 \\
\hline 6 & 18 & 590 & 551.58 & 553.29 & 518.93 & 600.36 \\
\hline 7 & 28 & 710 & 683.46 & 686.94 & 642.86 & 762.87 \\
\hline 8 & 28 & 910 & 877.42 & 820.92 & 816.70 & 889.79 \\
\hline 9 & 18 & 820 & 770.56 & 788.76 & 742.89 & 815.90 \\
\hline 10 & 18 & 560 & 484.13 & 516.45 & 528.86 & 529.86 \\
\hline 11 & 46 & 860 & 748.23 & 743.90 & 665.04 & 823.15 \\
\hline 12 & 28 & 850 & 867.41 & 846.44 & 865.12 & 855.07 \\
\hline 13 & 18 & 1150 & 1124.70 & 1116.50 & 1102.98 & 1122.48 \\
\hline 14 & 18 & 510 & 497.86 & 512.51 & 463.71 & 553.51 \\
\hline 15 & 12 & 320 & 315.35 & 332.07 & 345.39 & 253.85 \\
\hline 16 & 13 & 590 & 535.99 & 530.88 & 536.24 & 444.62 \\
\hline 17 & 14 & 420 & 404.35 & 387.22 & 374.14 & 332.12 \\
\hline 18 & 34 & 350 & 336.04 & 377.02 & 341.26 & 275.77 \\
\hline 19 & 44 & 350 & 337.06 & 232.99 & 356.47 & 256.38 \\
\hline 20 & 45 & 300 & 253.50 & 267.99 & 269.34 & 183.59 \\
\hline 21 & 18 & 590 & 585.79 & 566.60 & 565.69 & 564.61 \\
\hline 22 & 28 & 680 & 671.81 & 658.48 & 642.02 & 703.36 \\
\hline 23 & 11 & 490 & 571.48 & 519.61 & 467.67 & 537.27 \\
\hline 24 & 12 & 350 & 375.25 & 327.25 & 291.17 & 348.11 \\
\hline 25 & 13 & 460 & 431.31 & 472.40 & 439.37 & 499.80 \\
\hline 26 & 21 & 530 & 541.04 & 541.26 & 467.75 & 546.89 \\
\hline 27 & 22 & 270 & 249.97 & 256.29 & 246.75 & 295.24 \\
\hline 28 & 23 & 630 & 642.55 & 615.97 & 624.48 & 642.79 \\
\hline 29 & 28 & 790 & 644.69 & 690.69 & 667.52 & 612.60 \\
\hline 30 & 38 & 900 & 894.85 & 761.80 & 778.49 & 771.69 \\
\hline 31 & 38 & 930 & 964.89 & 997.30 & 951.00 & 953.70 \\
\hline 32 & 28 & 750 & 786.99 & 747.79 & 677.68 & 738.14 \\
\hline 33 & 28 & 710 & 587.19 & 672.03 & 589.65 & 745.66 \\
\hline 34 & 38 & 830 & 761.49 & 762.65 & 719.17 & 786.27 \\
\hline 35 & 48 & 720 & 670.95 & 689.62 & 649.22 & 693.04 \\
\hline 36 & 18 & 560 & 533.38 & 517.29 & 497.46 & 571.17 \\
\hline
\end{tabular}

Table 7 shows the measurements of the correlation coefficient between the measurement of volume obtained from the different windowing protocols and the true volume. The best windowing protocol was 1131/1858 for both observers. Tooth volumes obtained with manual segmentation of CBCT images and using the 1131/1858 windowing protocol were not significantly different from tooth volumes obtained by laser scan. 
Table 5. Intra-observer correlations for the 2 observers, in relation to the 4 different types of windowing protocols

\begin{tabular}{|c|c|c|c|}
\hline Observer & $\begin{array}{c}\text { Windowing } \\
\text { protocol } \\
\text { (level/width) }\end{array}$ & ICC & $\begin{array}{c}\text { p-value (paired Student's } \\
\text { t-test between first and } \\
\text { second measurements) }\end{array}$ \\
\hline \multirow{3}{*}{ Observer 1 } & AUTO & 0.964 & 0.56 \\
\hline & $1131 / 1858$ & 0.983 & 0.53 \\
\hline \multirow{3}{*}{ Observer 2 } & $2224 / 4095$ & 0.985 & 0.15 \\
& $1131 / 4095$ & 0.979 & 0.37 \\
& AUTO & 0.983 & 0.23 \\
\hline & $1131 / 1858$ & 0.934 & 0.0073 \\
\hline
\end{tabular}

ICC - two-way random, absolute agreement; $p$-value is significant if $p<0.05 / 8$ (because there were 8 tests) $=p<0.00625$.

Table 6. Inter-observer correlations for the 2 observers, in relation to the 4 different types of windowing protocols

\begin{tabular}{|l|c|c|}
$\begin{array}{c}\text { Windowing } \\
\text { protocol }\end{array}$ & ICC & $\begin{array}{c}\text { p-value (paired Student's t-test between } \\
\text { measurements by observers 1 and 2) }\end{array}$ \\
\hline AUTO & 0.986 & $<0.0001^{*}$ \\
\hline $1131 / 1858$ & 0.986 & 0.24 \\
$2224 / 4095$ & 0.986 & $0.0004^{*}$ \\
$1131 / 4095$ & 0.991 & 0.04 \\
\hline
\end{tabular}

* $p$-value is significant if $p<0.05 / 4$ (because there were 4 tests) $=p<0.0125$.

Table 7. Measurements of correlation coefficients between the measurements of volume obtained using different windowing protocols and the true volume from the laser scanner

\begin{tabular}{|c|c|c|c|c|}
\hline \multirow{2}{*}{ Observer } & $\begin{array}{c}\text { Windowing } \\
\text { protocol }\end{array}$ & $\begin{array}{c}\text { Mean } \\
\text { difference }\end{array}$ & p-value & $\begin{array}{c}\text { Correlation of } \\
\text { coefficient r }\end{array}$ \\
\hline \multirow{3}{*}{ Observer 1 } & AUTO & -50.7 & $<0.0001$ & 0.988 \\
\hline & $1131 / 1858$ & 13.3 & 0.048 & 0.983 \\
\hline \multirow{3}{*}{ Observer 2 } & $2224 / 4095$ & 35.85 & $<0.0001$ & 0.986 \\
\hline & $1131 / 4095$ & 28.29 & $<0.0001$ & 0.983 \\
\hline & AUTO & -74.491 & $<0.001$ & 0.979 \\
\hline & $1131 / 1858$ & 19.95 & 0.0012 & 0.987 \\
\hline
\end{tabular}

\section{Discussion}

Pauwels et al. stated in 2015 that the quantitative use of grey values should be avoided in CBCT imaging, as grey values are unreliable. ${ }^{20}$ Grey values measured on CBCT are dependent on the type of CBCT device, the exposure parameters, the position of the measurement inside the field of view, and the amount of mass inside and outside the field of view. ${ }^{20}$ The lack of consistent grey values means that a threshold value for bone and other tissues cannot be established for CBCT images. ${ }^{20}$ However, a modified greyvalue approach has also been investigated, with positive results in vitro and in vivo in terms of bone quality measurements and implant planning. ${ }^{21-24}$ In this study we did not measure grey values in CBCT; we only tested different windowing protocols for $\mathrm{CBCT}$ images to find the best contrast between teeth and the surrounding structures. We found that a protocol proposed (but not validated) by Spin-Neto for I-CAT (1131/1858) was significantly the best for obtaining accurate tooth volumes from in vivo I-CAT CBCT images (Fig. 2). ${ }^{17}$ Moreover, during manual segmentation, we encountered various parameters that may directly affect the qualitative and quantitative aspects of manual segmentation. These parameters may explain why the measurements were different between the 2 observers, and why the measurements were different when using different windowing protocols. These parameters could be categorized as: 1 . parameters specific to the volume to be segmented, 2. parameters specific to the observer, and 3. parameters associated with the segmentation software. Parameters that were specific to the volume to be segmented were 1. the anatomy of the tooth and of adjacent periodontal structures, 2 . the position of the tooth in the surrounding bone, 3 . the level of mineralization of the tooth, 4. the presence of metallic artifacts, and 5 . contact between adjacent teeth. The anatomy and density of a tooth is heterogeneous from the crown to the apex. A tooth crown is easier to segment due to its mineralized enamel and because the enamel presents a clear contrast with the adjacent air. The root and especially the apex are areas that are more complex to delineate by segmentation, due to the less clear difference between root tissue and the surrounding alveolar bone. Moreover, a disease-free periodontal ligament (lamina dura) cannot be distinguished on CBCT images with the field of view we used in our study, and may be mistaken for the tooth, in which case the final volume would be overestimated (Fig. 3). When we consider the position of the tooth in relation to the surrounding bone, segmentation between the tooth and bone becomes more difficult when the roots are closer to the cortical bone. This occurs more often on the mandible, where the teeth are positioned between the thin internal and external cortical bone, compared to the maxilla (Fig. 4).

Problems inherent to the mineralization of the tooth, such as the presence of decay and immature roots, may also complicate segmentation. For this reason, one of the initial exclusion criteria was the presence of major decay

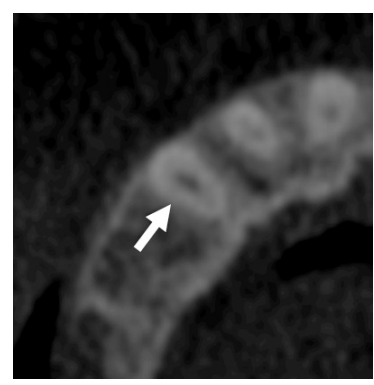

Fig. 3. The impossibility of discerning between the periodontal ligament and tooth (arrow). Windowing protocol 2224/4095 


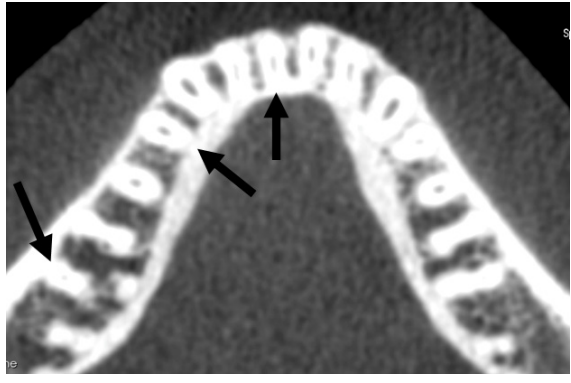

Fig. 4. AUTO

windowing protocol Fusion of the image of the tooth with the adjacent cortical bone (arrows)

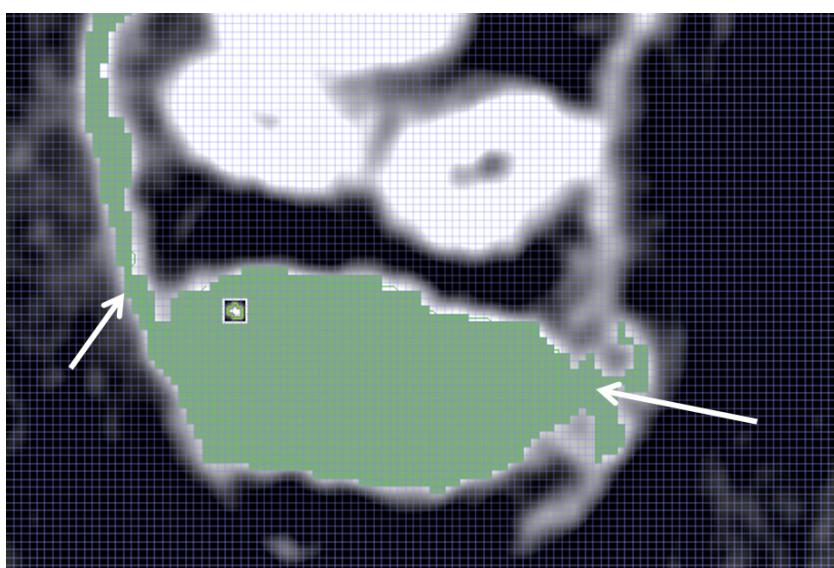

Fig. 5. Manual segmentation (green) of tooth number 18. Level tracing tool errors in selecting surrounding bony structures independent of the tooth (arrows)

that was clinically diagnosed prior the tooth removal. Immature roots present with low density in the apex region, and the limits of the apex in relation to the surrounding bone become difficult to determine. ${ }^{25}$ Metallic crowns and dental fillings create major artifacts in 3D tooth reconstruction and prevent accurate segmentation of crowns (Fig. 1). For this reason, we also excluded all teeth with crowns and metallic fillings from our study. Inter-dental contact makes segmentation and the interpretation of the external limits of dental crowns difficult. The teeth appear to be merged together on 2D slices.

Parameters specific to the observer that may affect the results of segmentation include: 1 . the observer's experience using segmentation software; 2 . his/her knowledge of dental anatomy, which is important when the observer needs to contour the apex region with complex root anatomy; and 3. any tiredness on the observer's part during the manual segmentation process, which may decrease the accuracy of segmentation, particularly with complex root anatomy, and when the time spent on one segmentation is excessively long.

Parameters specific to the software used (3D Slicer) are 1. the tools used for manual segmentation, and 2. the contrast/windowing control. We mainly used the "paint", "draw" and "level tracing" tools in the 3D Slicer software. The "level tracing" tool is well suited for tooth segmentation with clear limits, but if the limits are not precise or if there is a structure with a density close to that of the tooth, the "level tracing" tool is not the best option (Fig. 5).
Other parameters that may modify the results of manual segmentation are related to the CBCT radiological protocol, such as $\mathrm{mAs}, \mathrm{kVp}$, slice thickness, field of view and voxel size, and also due to movement artifacts in the CBCT device. ${ }^{26,27}$

The limitations of this study are related to the CBCT radiological protocol. We tested the windowing protocols in only one type of radiological protocol, without modification of the type of CBCT device, mAs, $\mathrm{kVp}$, slice thickness, field of view or voxel size. We also did not compare windowing protocol results between different types of CBCT devices. Therefore, our findings are strictly limited to the use of 3D Slicer software and to I-CAT CBCT, with a $0.3 \mathrm{~mm}$ slice thickness, $8 \mathrm{~cm} \times 16 \mathrm{~cm}$ field of view, $120 \mathrm{kVp}$ and $18 \mathrm{mAs}$. Also, we used only manual segmentation, as this technique still represents a gold standard. However, other in vitro teeth segmentation methods that have already been tested, provide much faster segmentation and are useful in clinical practice. ${ }^{28-30}$

In conclusion, the null hypothesis was rejected. Compared with the other windowing protocols we investigated, the 1131/1858 windowing protocol allows for significantly closer $3 \mathrm{D}$ in vivo segmentation of a tooth from I-CAT CBCT, with excellent intra-observer repeatability and inter-observer reproducibility.

\section{References}

1. Yezdani AA. Transparent aligners: An invisible approach to correct mild skeletal class III malocclusion. J Pharm Bioallied Sci. 2015;7: S301-S306.

2. Li W, Chen F, Zhang F, et al. Volumetric measurement of root resorption following molar mini-screw implant intrusion using cone beam computed tomography. PLoS One. 2013;8:e60962. doi:10.1371/journal.pone.0060962

3. Kalra S, Tripathi T, Rai P, Kanase A. Evaluation of orthodontic miniimplant placement: A CBCT study. Prog Orthod. 2014;15:61. doi:10. 1186/s40510-014-0061-x

4. Morea C, Hayek JE, Oleskovicz C, Dominguez GC, Chilvarquer I. Precise insertion of orthodontic miniscrews with a stereolithographic surgical guide based on cone beam computed tomography data: A pilot study. Int J Oral Maxillofac Implants. 2011;26:860-865.

5. Shahbazian M, Jacobs R, Wyatt J, et al. Validation of the cone beam computed tomography-based stereolithographic surgical guide aiding autotransplantation of teeth: Clinical case-control study. Oral Surg Oral Med Oral Pathol Oral Radiol. 2013;115:667-675.

6. Khalil W, EzEldeen M, Van De Casteele E, et al. Validation of cone beam computed tomography-based tooth printing using different three-dimensional printing technologies. Oral Surg Oral Med Oral Pathol Oral Radiol. 2016;121:307-315.

7. Anssari Moin D, Hassan B, Wismeijer D. A novel approach for custom three-dimensional printing of a zirconia root analogue implant by digital light processing. Clin Oral Implants Res. 2016;25. doi:10.1111/ clr.12859

8. Pirker W, Kocher A. Root analog zirconia implants: True anatomical design for molar replacement - a case report. Int J Periodontics Restorative Dent. 2011;31:663-668.

9. Vandevoort FM, Bergmans L, Van Cleynenbreugel J, et al. Age calculation using $X$-ray microfocus computed tomographical scanning of teeth: A pilot study. J Forensic Sci. 2004;49:787-790.

10. Liu Y, Olszewski R, Alexandroni ES, Enciso R, Xu T, Mah JK. The validity of in vivo tooth volume determinations from cone-beam computed tomography. Angle Orthod. 2010;80:160-166.

11. Wang Y, He S, Yu L, Li J, Chen S. Accuracy of volumetric measurement of teeth in vivo based on cone beam computer tomography. Orthod Craniofac Res. 2011;14:206-212. 
12. Koch GG. Intraclass correlation coefficient. In: Kotz S, and Johnson NL, ed. Encyclopedia of Statistical Sciences. $4^{\text {th }}$ ed. New York, NY: John Wiley \& Sons; 1982:213-217.

13. Forst D, Nijjar S, Flores-Mir C, Carey J, Secanell M, Lagravere M. Comparison of in vivo 3D cone-beam computed tomography tooth volume measurement protocols. Prog Orthod. 2014;15:69. doi:10.1186/ s40510-014-0069-2

14. Aizawa M, Nishikawa K, Sasaki K, et al. Automatic segmentation of teeth from dentomaxillofacial 3D-CT images. Nihon Hoshasen Gijutsu Gakkai Zasshi. 2010;66:343-352.

15. Le BH, Deng Z, Xia J, Chang YB, Zhou X. An interactive geometric technique for upper and lower teeth segmentation. Med Image Comput Assist Interv. 2009;12:968-975.

16. Zhao M, Ma L, Tan W, Nie D. Interactive tooth segmentation of dental models. Conf Proc IEEE Eng Med Biol Soc. 2005;1:654-657.

17. Spin-Neto R, Marcantonio E Jr, Gotfredsen E, Wenzel A. Exploring CBCT-based DICOM files. A systematic review on the properties of images used to evaluate maxillofacial bone grafts. J Digit Imaging. 2011;24:959-966.

18. Lee RJ, Pham J, Choy M, el al. Monitoring of typodont root movement via crown superimposition of single cone-beam computed tomography and consecutive intraoral scans. Am J Orthod Dentofacial Orthop. 2014;145:399-409.

19. Fedorov A, Beichel R, Kalpathy-Cramer J, et al. 3D Slicer as an Image Computing Platform for the Quantitative Imaging Network. Magn Reson Imaging. 2012;30:1323-1341.

20. Pauwels R, Jacobs R, Singer SR, Mupparapu M. CBCT-based bone quality assessment: Are Hounsfield units applicable? Dentomaxillofac Radiol. 2015;44:20140238. doi:10.1259/dmfr.20140238
21. Bujtár P, Simonovics J, Zombori G, et al. Internal or in-scan validation: A method to assess CBCT and MSCT gray scales using a human cadaver. Oral Surg Oral Med Oral Pathol Oral Radiol. 2014;117:768-779.

22. Andruch K, Płachta A. Evaluating maxilla bone quality through clinical investigation of voxel grey scale values from cone-beam computed tomography for dental use. Adv Clin Exp Med. 2015;24:1071-1077.

23. Valiyaparambil JV, Yamany I, Ortiz D, et al. Bone quality evaluation: Comparison of cone beam computed tomography and subjective surgical assessment. Int J Oral Maxillofac Implants. 2012;27:1271-1277.

24. Parsa A, Ibrahim N, Hassan B, Motroni A, van der Stelt P, Wismeijer D. Reliability of voxel gray values in cone beam computed tomography for preoperative implant planning assessment. Int J Oral Maxillofac Implants. 2012;27:1438-1442

25. Maret D, Molinier F, Braga J, et al. Accuracy of 3D reconstructions based on cone beam computed tomography. J Dent Res. 2010;89: 1465-1469.

26. Al-Rawi B, Hassan B, Vandenberge B, Jacobs R. Accuracy assessment of three-dimensional surface reconstructions of teeth from cone beam computed tomography scans. JOral Rehabil. 2010;37:352-358.

27. Ye N, Jian F, Xue J, et al. Accuracy of in-vitro tooth volumetric measurements from cone-beam computed tomography. Am J Orthod Dentofacial Orthop. 2012;142:879-887.

28. Na SD, Lee G, Lee JH, Kim MN. Individual tooth region segmentation using modified watershed algorithm with morphological characteristic. Biomed Mater Eng. 2014;24:3303-3309.

29. Ji DX, Ong SH, Foong KW. A level-set based approach for anterior teeth segmentation in cone beam computed tomography images. Comput Biol Med. 2014;50:116-128.

30. Gan Y, Xia Z, Xiong J, Zhao Q, Hu Y, Zhang J. Toward accurate tooth segmentation from computed tomography images using a hybrid level set model. Med Phys. 2015;42:14-27. 